\title{
Simulations of gamma-ray narrow-line Seyfert 1 galaxies with the Cherenkov Telescope Array
}

\author{
P. Romano*, ${ }^{a}$ S. Vercellone,${ }^{a}$ F. Tavecchio, ${ }^{a}$ M. Landoni, ${ }^{a}$ J. Knödlseder,${ }^{b}$ for the CTA \\ Consortium, L. Foschini, ${ }^{a}$
}

a INAF, Osservatorio Astronomico di Brera, Via E. Bianchi 46, I-23807, Merate, Italy

${ }^{b}$ Institut de Recherche en Astrophysique et Planétologie, 9 avenue Colonel-Roche, 31028,

Toulouse Cedex 4, France

E-mail: patrizia.romano@brera.inaf.it

\begin{abstract}
Gamma-ray emitting narrow-line Seyfert 1 ( $\gamma$-NLSy1) galaxies are jetted sources hosting a relatively low-mass black hole $\left(10^{6}-10^{8} M_{\odot}\right)$ which accretes close to the Eddington limit. $\gamma$-NLSy1 galaxies show flux and spectral variability in the gamma-ray energy band and radio properties similar to those of blazars, which indicate the presence of a relativistic jet. These properties make them interesting to investigate with the Cherenkov Telescope Array (CTA), the next-generation ground-based gamma-ray observatory. CTA will cover the $20 \mathrm{GeV}-300 \mathrm{TeV}$ energy range, with an improvement in average differential sensitivity by a factor 5-20 with respect to the current imaging atmospheric Cherenkov telescope arrays. For transients/flaring events (timescales of $\sim 1$ day or shorter) CTA will be about two orders of magnitude more sensitive with respect to FermiLAT at the overlapping energy of $25 \mathrm{GeV}$, allowing an unprecedented opportunity to investigate flaring $\gamma$-NLSy1 galaxies. We present preliminary results obtained by simulating the prototypical $\gamma$-NLSy1 galaxy, PMN J0948+0022, by means of the CTA public ctools software and the public instrument response files, investigating its possible detection and spectral properties, taking into account the effect of both the extra-galactic background light and intrinsic absorption components.
\end{abstract}

Revisiting narrow-line Seyfert 1 galaxies and their place in the Universe - NLS1 Padova 9-13 April 2018

Padova Botanical Garden, Italy

\footnotetext{
* Speaker.
} 


\section{Introduction}

In the last decade evidence has been accumulating that suggests that NLS1s may host a relativistic jet, as blazars do. Indeed, although historically considered hosted in spiral/barred galaxies [1], and generally not strong radio emitters, $4-7 \%$ of the NLS1 population have turned out to be radio loud $[2,3]$ and with a flat radio spectrum [4-6].

The smoking gun was the first detection of PMN J0948+0022 [7, 8], by Fermi-LAT in the high-energy $\gamma$ band $(\mathrm{E}>100 \mathrm{MeV})$, closely followed by the discovery that its multi-wavelength behaviour was that of a source with a relativistic jet, like those observed in blazars $[9,10]$. At the time of writing Fermi-LAT has found a total of 19 NLS1s in $\gamma$-rays. Still, no firm detection has been obtained in the very high energy (VHE) regime: Whipple did not detect significant emission from 1H 0323+342 above $400 \mathrm{GeV}$ [11], and VERITAS observations of PMN J0948+0022 (5 hr) only yielded upper limits at $\mathrm{E}>100 \mathrm{GeV}$ [12].

Detecting high-energy ( $\mathrm{E}$ in excess of a few tens of $\mathrm{GeV}$ ) emission from NLS1 galaxies is challenging for several reasons. First, they have on average rather soft spectra $(\Gamma \sim 2.5)$. Second, some of them have a rather high redshift $(z>0.3)$ so we expect a large absorption by extragalactic background light (EBL). Furthermore, if broad-line region absorption is present, it could produce a spectral break/cut-off at energies of $20-30 \mathrm{GeV}$, further weakening the high-energy spectrum.

On the other hand, some NLS1s exhibit strong gamma-ray flares, on timescales in the order of a few hours/a day in $\gamma$-rays, as observed, for instance in PMN J0948+0022 [10, 12], which is considered a prototypical object. Furthermore, the high-state activity can last for several weeks/months, repeated on a multi-year baseline (e.g. SBS 0846+513 [13]).

Given these properties, we started an exploratory investigation of the prospects for observation of NLS1s with the Cherenkov Telescope Array (CTA), the next-generation ground-based gammaray observatory. In this paper we report the preliminary results on one of the 19 sources that constitute the complete $\gamma$-NLS1 sample, PMN J0948+0022. The full set of simulations will be presented in a forthcoming paper (Romano et al., in prep.).

\section{CTA}

The Cherenkov telescope array (CTA) [14 - 17], the first open VHE observatory, will operate on two sites to provide all-sky coverage; the Northern site will be located at the Observatorio del Roque de los Muchachos on the island of La Palma (Spain), and the Southern site, at the European Southern Observatory's (ESO's) Paranal Observatory in the Atacama Desert (Chile).

The combination of different classes of telescopes, i.e., the large-sized telescopes (LSTs, diameter D 23 m, 20-150 GeV), the medium-sized telescopes (MSTs, D 12 m, 0.15-5 TeV), and the small-sized telescopes (SSTs, primary mirror D 4 m, $5 \mathrm{TeV}-300 \mathrm{TeV}$ ), will provide a broad $(20 \mathrm{GeV}-300 \mathrm{TeV})$ energy coverage.

The average differential sensitivity will be a factor 5-20 better with respect to the current imaging atmospheric Cherenkov telescope (IACT) arrays. A marked improvement will also be the extension of the accessible energy range from well below $100 \mathrm{GeV}$ to above $100 \mathrm{TeV}$ [18], the former being a region of utmost interest for NLS1s. 

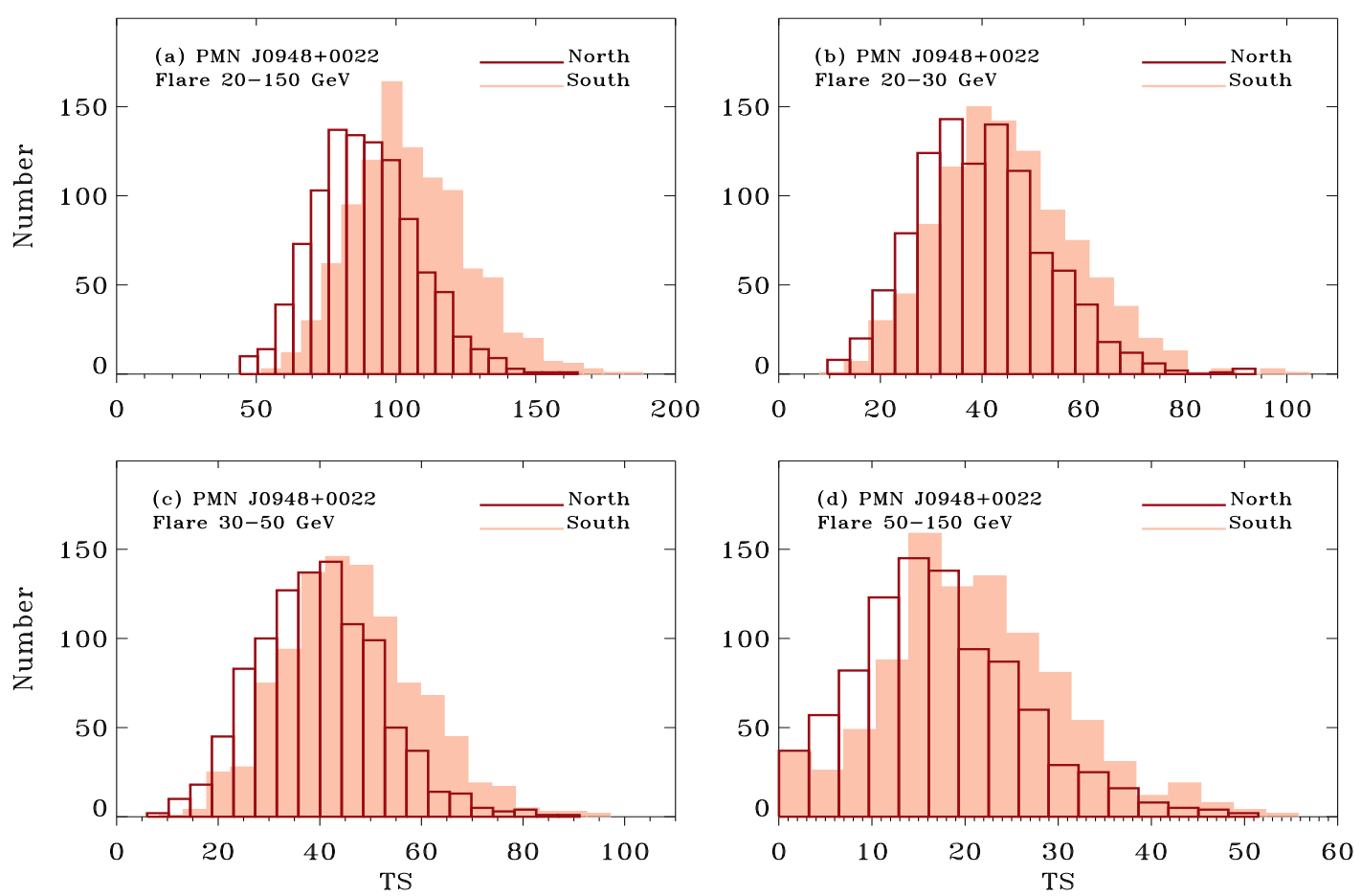

Figure 1: TS value distributions for several energy bands for PMN J0948+0022 in the flaring state (10 hr exposure).

CTA will offer a huge advantage over Fermi in the energy range of overlap for $\sim$ minute to $\sim$ day timescale phenomena [19] such as explosive transients, AGN flares, and binary systems (although it will also have the disadvantage of a limited field of view, which can be partially circumvented by a prompt reaction to external triggers).

\section{CTA Simulations}

We performed our simulations with the ctools (v1.4.2) public analysis package [20 $]^{1}$ and the public CTA instrument response files ${ }^{2}$ (IRF, v. prod3b-v1). We chose the IRFs based on the source visibility from both CTA sites (with a zenith angle $\sim 20^{\circ}$ ) and with analysis cuts optimized for exposures comparable to both the source variability timescales and CTA long term observing plan.

The basic input of our simulations is the spectrum of the source, derived as an extrapolation of the best-fit Fermi spectra to the CTA energy range, including the effects of the $\gamma$-ray absorption both along the path to the Earth (EBL) [21], and inside the source (internal absorption) [22], which was modelled by a cut-off at $\sim 30 \mathrm{GeV}$. We considered three gamma-ray states:

- quiescence, described by a broken power law [7] with a break at $E_{\mathrm{b}}=1 \mathrm{GeV}$, photon indices $\Gamma_{1}=2.3$ and $\Gamma_{2}=3.4$, and $F_{\mathrm{E}>200 \mathrm{MeV}}=(3.9 \pm 0.3) \times 10^{-8} \mathrm{ph} \mathrm{cm}^{-2} \mathrm{~s}^{-1}$;

\footnotetext{
${ }^{1}$ http://cta.irap.omp.eu/ctools/.

${ }^{2}$ https://www.cta-observatory.org/science/cta-performance/.
} 

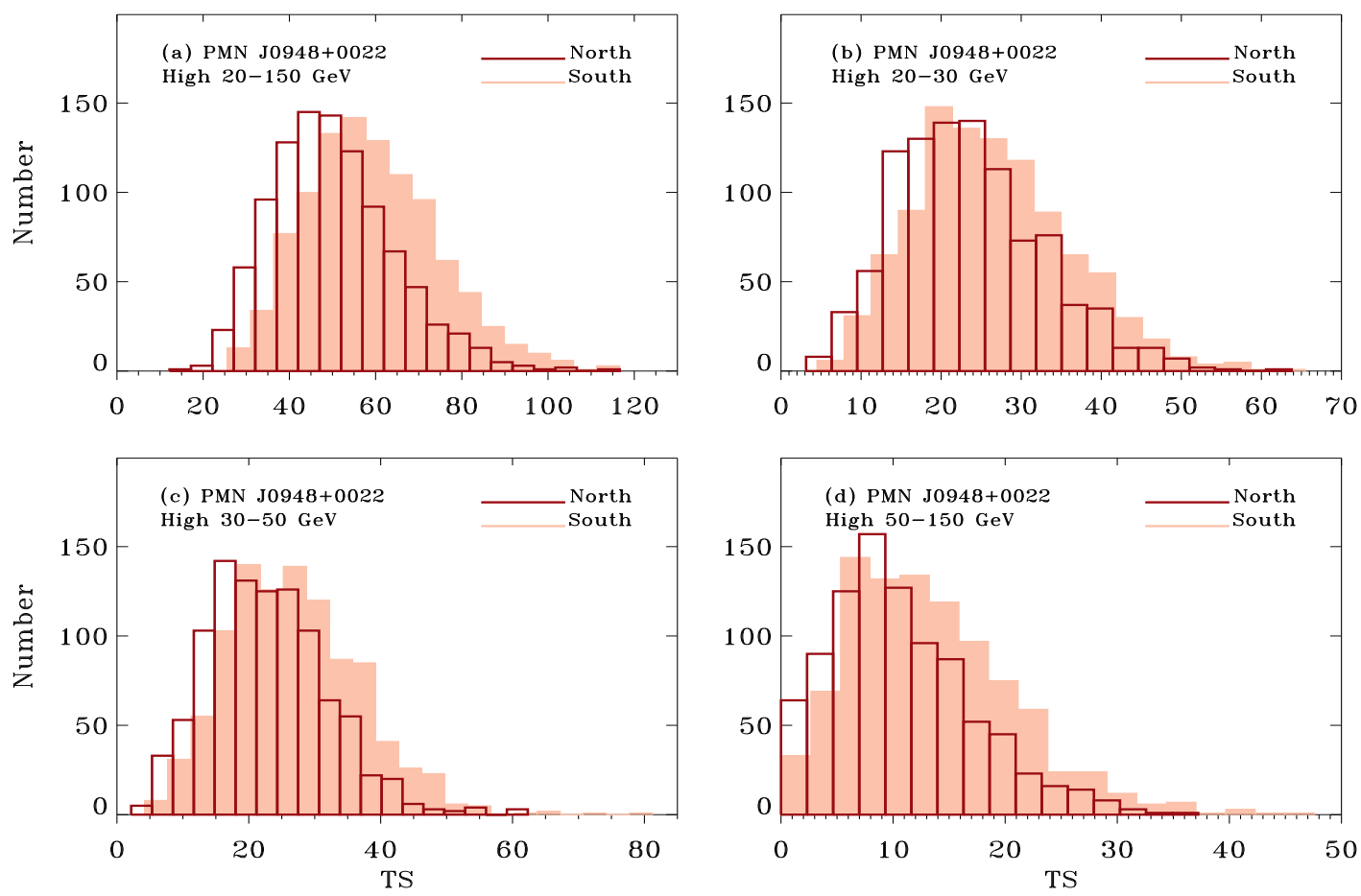

Figure 2: TS value distributions for several energy bands for PMN J0948+0022 in the high state $(50 \mathrm{hr}$ exposure).

- a high-state, described by a power law [10] with $\Gamma=2.55$, and $F_{\mathrm{E}>100 \mathrm{MeV}}=(1.02 \pm 0.02) \times$ $10^{-6} \mathrm{ph} \mathrm{cm}^{-2} \mathrm{~s}^{-1}$

- a flaring-state, not observed in reality but assumed to be 3 times brighter than the high-state of [10].

For each source state and CTA site, we performed 1000 statistically independent realisations by adopting different seeds for the background randomization to reduce the impact of variations between individual realisations. We consider a source detected with a high significance when the test statistic (TS, [23],[24]) of the maximum likelihood model fitting is such that $T S \geq 25$, with a low significance when $10 \leq T S<25$, and not detected for $T S<10$. For each simulation (set of 1000 realisations) the mean TS value and its uncertainty were calculated as the mean, $\overline{T S_{\text {sim }}}=\frac{1}{N} \sum_{k=1}^{N} T S_{\text {sim }}(k)$, and square root of the standard deviation of the sample of $N$ values, $s_{\text {sim }}^{2}=\frac{1}{N-1} \sum_{k=1}^{N}\left(T S_{\text {sim }}(k)-\overline{T S_{\text {sim }}}\right)^{2}$.

\section{Results and conclusions}

We started an exploratory investigation on $\gamma$-NLS1 galaxies with CTA, and hereon we present the preliminary results on the prototype of the class, PMN J0948+0022. In a forthcoming paper (Romano et al., in prep.) we shall report on the remainder of the sample and investigate the detectability with different models based on different locations of the $\gamma$-ray emitting region.

As an example of our methodology, in Fig. 1 we show the distributions of the TS values for several energy bands for PMN J0948+0022 in the flaring state when simulated with a $10 \mathrm{hr}$ 

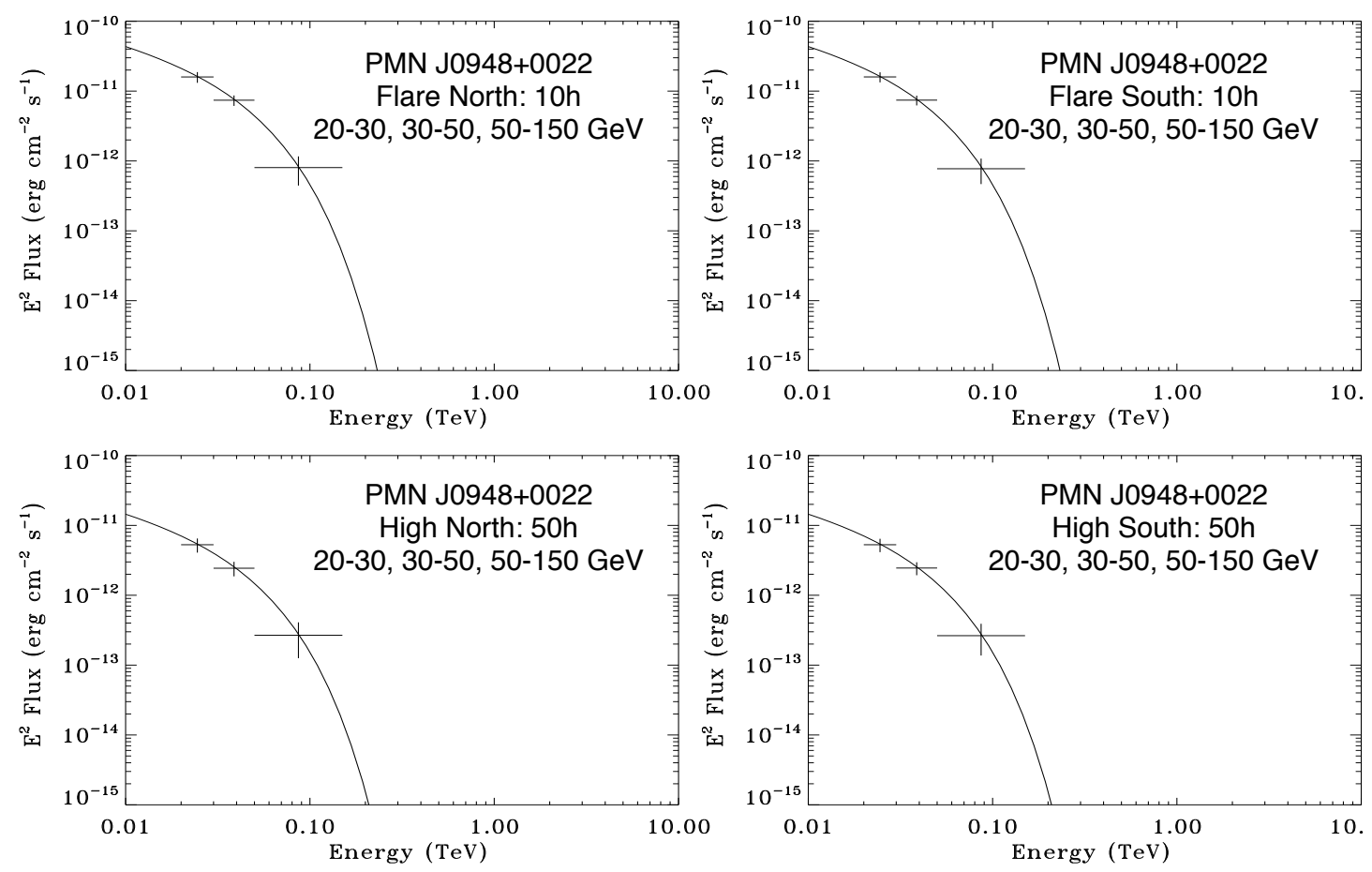

Figure 3: Spectral energy distributions of PMN J0948+0022 in the flaring and high state from both CTA sites. The black line represent the input model after EBL and internal absorption, the points are the simulated fluxes.

exposure, corresponding roughly to 2-3 days of CTA observations. Fig. 2, instead, shows the TS distributions for the high state when simulated with a $50 \mathrm{hr}$ exposure (corresponding to the typical observation time for a guest observer proposal on a given source within one year).

Fluxes (and uncertainties) in each band were calculated as the mean (and square root of the standard deviation) of the sample of 1000 values. From those values we produced the spectral energy distributions (SED). Figure 3, for instance, shows the SEDs in the flaring and high state.

To summarise our results, we find that

- when PMN J0948+0022 is in the "flare" state it is well detected in all bands in $10 \mathrm{hr}$ (with a TS mean value ranging from $\sim 17$ to $\sim 46$ depending on the energy band, and TS of $\sim 105$ for the $20-150 \mathrm{GeV}$ band); it is also detected in all bands up to $50 \mathrm{GeV}$ in only $3 \mathrm{hr}$ (TS> 13);

- when the source is in the high state, it is detected in all bands in $50 \mathrm{hr}$ (with a TS mean value ranging from $\sim 11$ to $\sim 27$ depending on the energy band, and TS of $\sim 60$ for the $20-150 \mathrm{GeV}$ band); it is also detected in all bands up to $50 \mathrm{GeV}$ in $10 \mathrm{hr}$ (TS $>10$ ).

- PMN J0948+0022 is also detected in quiescence in the $20-150 \mathrm{GeV}$ and $20-50 \mathrm{GeV}$ band in $100 \mathrm{hr}$ (TS> 21 and TS $>14$, respectively).

Therefore, a high confidence detection can be obtained below $50 \mathrm{GeV}$ and given the characteristics of the spectra of these sources, the LSTs, and partly the MSTs, may be considered as the most promising telescopes for this investigation. In the highest energy range $(50-150 \mathrm{GeV})$, on the other hand, the EBL and the internal absorption play a relevant role. 
The harder-when-brighter behaviour observed in the Fermi-LAT data of the blazar PMN J0948+0022 may favour detections with CTA during flaring states when these source will be easier to detect, allowing us to constrain the location of the $\gamma$-ray emission region by comparing different competing spectral models.

If, as discovered for blazars, the location of the gamma-ray emitting region is not always at the same distance from the central black-hole during different flaring episodes of the same source [25, 26], then a detection of NLS1 galaxies at energies above $100 \mathrm{GeV}$ may help put tight constraints throughout each of these flares.

\section{Acknowledgments}

The authors acknowledge contribution from the grant INAF CTA-SKA, "Probing particle acceleration and $\gamma$-ray propagation with CTA and its precursors" (PI F. Tavecchio).

This research made use of ctools, a community-developed analysis package for Imaging Air Cherenkov Telescope data. ctools is based on GammaLib, a community-developed toolbox for the high-level analysis of astronomical gamma-ray data. This research has made use of the CTA instrument response functions provided by the CTA Consortium and Observatory, see https://www.ctaobservatory.org/science/cta-performance/ (version prod3b-v1) for more details.

We gratefully acknowledge financial support from the agencies and organizations listed here: http://www.cta-observatory.org/consortium_acknowledgments.

This paper went through internal review by the CTA Consortium.

This conference has been organized with the support of the Department of Physics and Astronomy "Galileo Galilei", the University of Padova, the National Institute of Astrophysics INAF, the Padova Planetarium, and the RadioNet consortium. RadioNet has received funding from the European Union's Horizon 2020 research and innovation programme under grant agreement No 730562.

\section{References}

[1] D. M. Crenshaw, S. B. Kraemer, and J. R. Gabel, AJ 126, 1690-1698 (2003).

[2] S. Komossa, W. Voges, D. Xu, S. Mathur, H.-M. Adorf, G. Lemson, W. J. Duschl, and D. Grupe, AJ 132, 531-545 (2006).

[3] V. Cracco, S. Ciroi, M. Berton, F. Di Mille, L. Foschini, G. La Mura, and P. Rafanelli, MNRAS 462, 1256-1280 (2016).

[4] A. Y. K. N. Oshlack, R. L. Webster, and M. T. Whiting, ApJ 558, 578-582 (2001).

[5] H.-Y. Zhou, T.-G. Wang, X.-B. Dong, Y.-Y. Zhou, and C. Li, ApJ 584, 147-152 (2003).

[6] W. Yuan, H. Y. Zhou, S. Komossa, X. B. Dong, T. G. Wang, H. L. Lu, and J. M. Bai, ApJ 685, 801-827 (2008).

[7] A. A. Abdo, M. Ackermann, M. Ajello, M. Axelsson, L. Baldini, et al., ApJ 699, 976-984 (2009).

[8] L. Foschini, Fermi/Lat Collaboration, G. Ghisellini, L. Maraschi, F. Tavecchio, and E. Angelakis, "Fermi/LAT Discovery of Gamma-Ray Emission from a Relativistic Jet in the Narrow-Line Seyfert 1 Quasar PMN J0948+0022," in Accretion and Ejection in AGN: a Global View, edited by L. Maraschi, G. Ghisellini, R. Della Ceca, and F. Tavecchio, 2010, vol. 427 of Astronomical Society of the Pacific Conference Series, pp. 243-248. 
[9] A. A. Abdo, M. Ackermann, M. Ajello, M. Axelsson, L. Baldini, et al., ApJ 707, 727-737 (2009).

[10] L. Foschini, G. Ghisellini, Y. Y. Kovalev, M. L. Lister, F. D’Ammando, et al., MNRAS 413, 1671-1677 (2011).

[11] A. D. Falcone, I. H. Bond, P. J. Boyle, S. M. Bradbury, J. H. Buckley, et al., ApJ 613, 710-715 (2004).

[12] F. D’Ammando, M. Orienti, J. Finke, C. M. Raiteri, T. Hovatta, et al., MNRAS 446, 2456-2467 (2015).

[13] V. S. Paliya, B. Rajput, C. S. Stalin, and S. B. Pandey, ApJ 819, 121 (2016).

[14] M. Actis, G. Agnetta, F. Aharonian, A. Akhperjanian, J. Aleksić, et al., Experimental Astronomy 32, 193-316 (2011).

[15] B. S. Acharya, M. Actis, T. Aghajani, G. Agnetta, J. Aguilar, F. Aharonian, M. Ajello, A. Akhperjanian, M. Alcubierre, J. Aleksić, and et al., Astroparticle Physics 43, 3-18 (2013).

[16] W. Hofmann, "The Cherenkov telescope array - Status," in 6th International Symposium on High Energy Gamma-Ray Astronomy, 2017, vol. 1792 of American Institute of Physics Conference Series, p. 020014.

[17] W. Hofmann, The Messenger 168, 21-26 (2017).

[18] The Cherenkov Telescope Array Consortium, :, B. S. Acharya, I. Agudo, I. A. Samarai, R. Alfaro, J. Alfaro, C. Alispach, R. Alves Batista, J.-P. Amans, and et al., ArXiv e-prints 1709.07997, (2017).

[19] S. Funk, J. A. Hinton, and CTA Consortium, Astroparticle Physics 43, 348-355 (2013).

[20] J. Knödlseder, M. Mayer, C. Deil, J.-B. Cayrou, E. Owen, et al., AEEA 593, A1 (2016).

[21] A. Domínguez, J. R. Primack, D. J. Rosario, F. Prada, R. C. Gilmore, et al., MNRAS 410, 2556-2578 (2011).

[22] J. Poutanen, and B. Stern, ApJL 717, L118-L121 (2010).

[23] W. Cash, ApJ 228, 939-947 (1979).

[24] J. R. Mattox, D. L. Bertsch, J. Chiang, B. L. Dingus, S. W. Digel, et al., ApJ 461, 396 (1996).

[25] L. Foschini, A. Treves, F. Tavecchio, D. Impiombato, G. Ghisellini, et al., AEAA 484, L35-L38 (2008).

[26] L. Foschini, G. Ghisellini, F. Tavecchio, G. Bonnoli, and A. Stamerra, ArXiv e-prints 1110.4471, 2011 Fermi Symposium proceedings - eConf C110509 (2011). 